\title{
Time to presentation and 12-month health outcomes in patients presenting to the emergency department with symptoms of possible acute coronary syndrome
}

\author{
Louise Cullen, ${ }^{1,2,3}$ Jaimi H Greenslade, ${ }^{1,2,3}$ Louven Menzies, ${ }^{1}$ Ashley Leong, ${ }^{3}$ \\ Martin Than ${ }^{4}$ Christopher Pemberton, ${ }^{5}$ Sally Aldous, ${ }^{4}$ John Pickering, ${ }^{4,5}$ \\ Emily Dalton, ${ }^{1}$ Bianca Crosling, ${ }^{6}$ Rachelle Foreman, ${ }^{6}$ William A Parsonage ${ }^{3,7}$
}

${ }^{1}$ Department of Emergency Medicine, Royal Brisbane and Women's Hospital, Brisbane, Queensland, Australia ${ }^{2}$ School of Public Health and Social Work, Queensland University of Technology, Brisbane, Queensland, Australia

${ }^{3}$ School of Medicine, The University of Queensland, Brisbane, Queensland, Australia

${ }^{4}$ Department of Emergency Medicine, Christchurch Hospital, Christchurch, New Zealand

${ }^{5}$ Department of Medicine, University of Otago, Christchurch, New Zealand ${ }^{6}$ National Heart Foundation, Melbourne, Australia ${ }^{7}$ Department of Cardiology, Royal Brisbane and Women's Hospital, Brisbane,

Queensland, Australia

\section{Correspondence to}

Dr Louise Cullen, Department of Emergency Medicine, Royal Brisbane and Women's Hospital, Ground Floor, Butterfield Street, Brisbane, QLD 4006, Australia; louise. cullen@health.qld.gov.au

Received 3 May 2015 Revised 3 December 2015 Accepted 6 December 2015 Published Online First 18 January 2016

\begin{abstract}
Objective To define the association between time taken to present to the emergency department (ED) with symptoms of possible acute coronary syndrome (ACS) and 1-year outcomes. We also determined whether particular patient characteristics are associated with delays in seeking care after symptom onset.

Methods We collected data, which included a customised case report form to record symptom onset, on adult patients presenting with suspected ACS to two EDs in Australia and New Zealand. Such patients were followed up prospectively for 1 year. The composite primary endpoint included death, acute myocardial infarction, unstable angina pectoris treated with revascularisation or readmission with heart failure occurring after discharge but within 12 months after the index presentation.
\end{abstract}

Results ACS was diagnosed at presentation in 420 $(16.8 \%)$ of 2515 patients recruited. Cox regression was conducted to assess the relationship between presentation time and the rate of primary endpoints after controlling for age, ethnicity, prior angina, prior coronary artery bypass graft and index diagnosis. Middle (2-6 h) and late presenters ( $>6 \mathrm{~h}$ postsymptom onset) developed the primary endpoint at a rate $1.22(95 \% \mathrm{Cl} 0.80$ to $1.85)$ and 1.57 (1.07 to 2.31) times higher than early presenters. Patients with known risk factors and cardiovascular disease were more likely to present late to the ED.

Conclusions There is an independent association between time to presentation and 1-year cardiac outcomes following initial chest pain assessment for ED patients with possible cardiac chest pain in the Australian and New Zealand setting. This association occurred irrespective of the eventual diagnosis. Effective public health campaigns and other measures that facilitate early presentation with symptoms for patients with symptoms suggestive of ACS are justified and may improve prognosis.

Trial registration number ACTRN12611001069943.

\section{CrossMark}

To cite: Cullen $\mathrm{L}$, Greenslade JH, Menzies L, et al. Emerg Med J 2016:33:390-395.

\section{INTRODUCTION}

For patients with symptoms suggestive of acute coronary syndrome (ACS), prehospital delay remains the largest component of the total time to management of patients with an acute myocardial infarction (AMI). ${ }^{1-4}$ The time taken to seek help for symptoms of ACS is higher for a number of patient groups,

\section{Key messages}

What is already known on this subject

- Suggestive of acute coronary syndrome, prehospital delay remains the largest component of the total time to management of patients with an acute myocardial infarction.

- For patients with ST-elevation myocardial infarction, patient delays portend poor prognosis.

\section{What might this study add?}

- Our study shows that there is an independent association between time to presentation and 1-year cardiac outcomes following initial chest pain assessment for ED patients with possible cardiac chest pain in the Australian and New Zealand setting.

- This association occurred irrespective of the eventual diagnosis.

- Effective public health campaigns and other measures that facilitate early presentation with symptoms suggestive of acute coronary syndrome are justified and may improve prognosis.

including patients who are of low socio-economic status, female sex, from a non-English speaking background and those travelling to the emergency department (ED) by private transport. ${ }^{5-11}$ Lack of education about cardiovascular disease also results in increased delays, with reduced time-to-presentation seen after focused education campaigns about the warning symptoms for heart disease. ${ }^{12} 13$

The management of AMI is well defined and includes timely diagnosis, ${ }^{2}$ expeditious angiography ${ }^{14}$ and the use of reperfusion strategies such as thrombolytic therapy ${ }^{15}{ }^{16}$ and percutaneous intervention (PCI). ${ }^{3}$ It is well established that delays in the management of AMI (particularly delays to reperfusion) correlate closely with adverse outcomes in patients with a ST-elevation myocardial infarction (STEMI), ${ }^{17}$ but for patients diagnosed with other ACSs (unstable angina and non-STEMI (NSTEMI)), the effect of prehospital delay is unclear. There may be no association between delayed medical care and mortality. ${ }^{18}$ Patients 
discharged from the ED with a diagnosis of non-cardiac chest pain have a reduced survival rate in comparison with agematched population rates, ${ }^{19}$ however there are no reports about whether delays to presentation in this cohort are associated with future cardiac events. A better understanding is needed about possible association between long-term cardiovascular outcomes and the time taken to seek assessment and treatment for the broader cohort of patients who present to ED with symptoms of possible ACS.

The primary aim of this study was to identify the relationship between the time to presentation with possible ACS and a composite endpoint including death, AMI, unstable angina treated with revascularisation or readmission with heart failure at 12 months after the index presentation. This was investigated in patients with and without an index diagnosis of ACS. The secondary aim was to determine whether particular patient characteristics are associated with a delay in seeking care after the onset of symptoms of possible ACS.

\section{METHODS}

\section{Design and setting}

Data from prospectively recruited patients with symptoms of possible ACS presenting to two large tertiary hospitals in Brisbane, Australia and Christchurch, New Zealand were analysed. $^{20}$ The hospital's ethics boards approved the study (HREC/14/QRBW/5) and all data were collected in accordance with ACTR no. ACTRN12611001069943.

Patients were enrolled between November 2007 and May 2013. Criteria for enrolment included age of 18 years or over with at least $5 \mathrm{~min}$ of symptoms consistent with ACS, where the attending physician planned to perform serial troponin ( $\mathrm{cTn}$ ) tests. The American Heart Association case definition for cardiac symptoms suggestive of acute ischaemia was used (ie, acute chest, epigastric, neck, jaw or arm pain; or discomfort or pressure without an apparent non-cardiac source). ${ }^{21}$ Patients were excluded for any of the following: a clear cause other than ACS for the symptoms (eg, examination findings of pneumonia), inability to provide informed consent, staff considered recruitment to be inappropriate (eg, terminal condition), transfer from another hospital, pregnancy, previous enrolment or inability to be contacted after discharge. Perceived high risk and STEMI at presentation were not exclusion criteria, however patients were excluded from the present analyses if their index admission was for heart failure only, they were without data on time to presentation or they did not consent to 1-year follow-up. Patients were managed according to local hospital protocols including blood draws for cTnI measurement at presentation, and then $6-12 \mathrm{~h}$ afterwards in compliance with international guidelines. ${ }^{21} 22$ Royal Brisbane and Women's Hospital used the DxI Access Accu cTnI assay (Beckman Coulter, Chaska, Minnesota, USA), detection limit of $10 \mathrm{ng} / \mathrm{L}$, 99th percentile of $40 \mathrm{ng} / \mathrm{L}, 10 \%$ coefficient of variation at $60 \mathrm{ng} / \mathrm{L}$ and decision cut-off as per

\section{Box 1 Composite primary endpoint}

Events occurring within 12 months of index presentation including:

- Acute myocardial infarction (non-ST-elevation myocardial infarction and ST-elevation myocardial infarction)

- Unstable angina pectoris treated with emergency or urgent revascularisation

- Admission for heart failure

manufacturer $>40 \mathrm{ng} / \mathrm{L}$. Christchurch Hospital used the Abbott ARCHITECT cTnI assay (Abbott, Chicago, Illinois, USA), detection limit of $<10 \mathrm{ng} / \mathrm{L}$, 99th percentile of $28 \mathrm{ng} / \mathrm{L}, 10 \%$ coefficient of variation at $32 \mathrm{ng} / \mathrm{L}$ and decision cut-off as per manufacturer $>30 \mathrm{ng} / \mathrm{L}$.

Data were collected prospectively by research nurses using a published data dictionary and included the time of onset of symptoms. ${ }^{23}$ Nursing staff collected demographic and clinical data from patients, supervised ECG testing and drew blood samples for cTnI testing. Patients were followed up at 30 days and 1 year to determine the occurrence of major adverse cardiac events as assessed by: (a) telephone contact by research staff, (b) review of patients' hospital notes and (c) a national health events search (identifying death) which was undertaken in January 2010 and July 2014 in Australia and May 2013 in New Zealand.

Thirty-day outcomes were adjudicated independently by one of two local cardiologists using predefined standardised reporting guidelines. Cardiologists had knowledge of the clinical record, ECG and troponin results from standard care. A second cardiologist conducted a blind review of all ACS cases and at least $10 \%$ of non-ACS cases. In cases of disagreement, endpoints were agreed by consensus. The composite primary endpoint (box 1) was AMI (NSTEMI and STEMI), unstable angina pectoris (UAP) treated with emergency or urgent revascularisation or admission for heart failure within 12 months. End points that occurred during the index hospital admission were not included (figure 1). Diagnosis of AMI was according to international guidelines and based on evidence of myocardial necrosis in the context of acute ischaemia. ${ }^{24}$ A diagnosis of UAP was based on ischaemic symptoms with troponin levels below the decision cut-off. Acute ischaemia was considered present based either on ischaemic ECG changes or results of subsequent investigation including exercise tolerance testing, myocardial perfusion scan, stress echocardiography suggestive of reversible ischaemia or angiography demonstrating significant obstructive coronary disease. ${ }^{24}$ This definition included patients with new symptoms or a changing symptom pattern (ie, from stable to unstable angina). Patients with equivocal ECG changes but clear positive changes on exercise tolerance testing or imaging evidence of

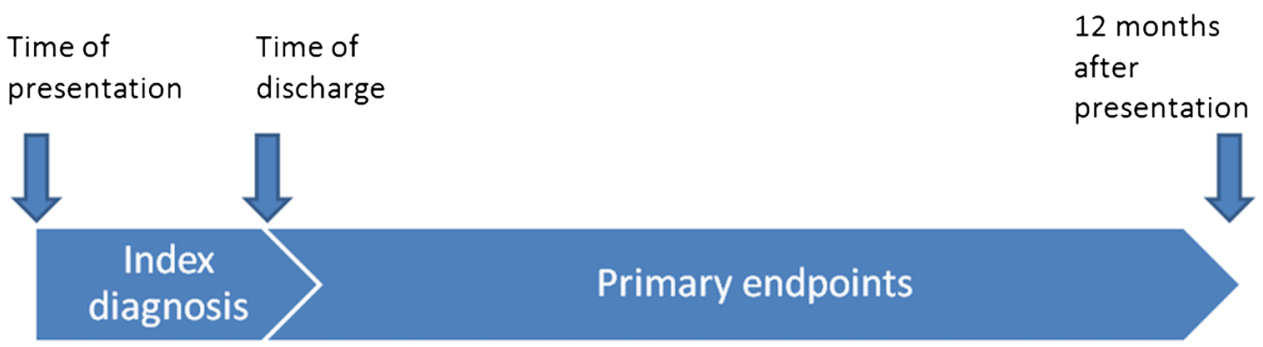

Figure 1 End points. 


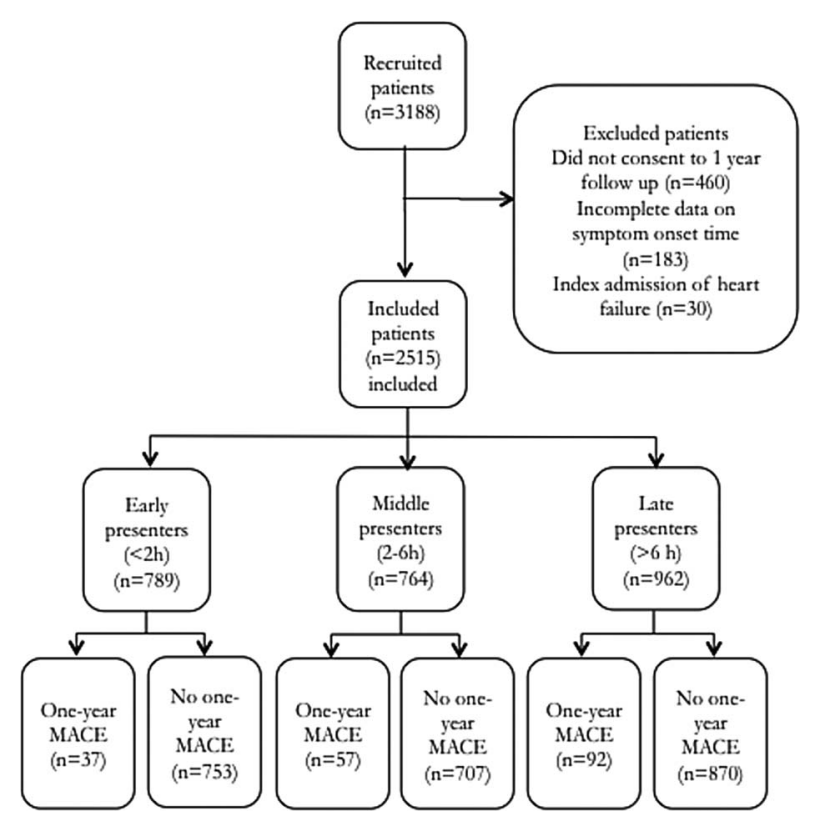

Figure 2 Participant flow. MACE, Major adverse cardiac event.

critical coronary stenosis were also classified as UAP. Only UAP that was treated with a revascularisation procedure was included in the primary endpoint. Outcomes for all patients who reported an event or readmission within 1 year were adjudicated by an independent emergency physician or cardiologist using data obtained from patient contact, hospital administrative data information and death registry information.

\section{Data collection and processing}

Data were analysed using Stata V.12 (StataCorp, 2011 College Station, Texas, USA). Presentation time was defined as the time interval (in hours) between the commencement of symptoms that precipitated the index presentation to ED and time of ED presentation. Patients were stratified as early, middle and late presenters if their presentation times were $<2 \mathrm{~h}, 2-6 \mathrm{~h}$ and $>6 \mathrm{~h}$ after onset of symptoms, respectively. This categorisation was chosen a priori. Baseline demographics and clinical characteristics were compared for early, middle and late presenters using standard descriptive statistics. The median time to ED presentation (and IQR) was reported for patients with index diagnoses of STEMI, NSTEMI, UAP treated with emergency or urgent revascularisation and non-cardiac chest pain.

Time to the primary endpoint was calculated for each individual and the log rank test was used to test the equality of the survival functions of the three delay intervals. Time to the primary endpoint was presented graphically using KaplanMeier failure functions following the recommendation of Pocock, Clayton and Altman. ${ }^{25}$ Cox proportional hazards regression was conducted to identify whether time to presentation was associated with primary endpoint rates after controlling for the baseline covariates that differed at $\mathrm{p}<0.1$ across early, middle and late presenters. Examination of the data indicated that the relationship between age and primary endpoint was curvilinear, therefore age was grouped into 10 -year categories and entered as a categorical variable into the Cox model.

Subgroup analyses were also conducted comparing patients whose diagnosis during the index presentation was ACS (AMI and UAP treated with emergency or urgent revascularisation) with those whose index diagnosis was not ACS. For each subgroup the association between time to presentation and primary endpoint was examined and a Cox regression analysis conducted. To exclude the known effect of time to reperfusion on prognosis in patients with STEMI, analyses were repeated for the ACS group with index STEMI excluded. The Cox models were assessed for the proportional hazards assumption and this was met in all instances.

Table 1 Demographic characteristics of the sample by arrival time

\begin{tabular}{|c|c|c|c|c|}
\hline Variable & $\begin{array}{l}\text { Early presenters } \\
(<2 \mathrm{~h})(\mathrm{n}=789)\end{array}$ & $\begin{array}{l}\text { Middle presenters } \\
(2-6 h)(n=764)\end{array}$ & $\begin{array}{l}\text { Late presenters } \\
(>6 \text { h) }(n=962)\end{array}$ & p Value \\
\hline Mean age, years (SD) & $56(14)$ & $58(15)$ & $59(15)$ & $<0.01$ \\
\hline Male sex & $480(60.8)$ & $443(58.0)$ & $576(60.0)$ & 0.51 \\
\hline Caucasian & 701 (91.5) & $649(87.4)$ & $809(86.2)$ & $<0.01$ \\
\hline Index AMI or urgent revascularisation & $102(12.9)$ & $122(16.0)$ & $196(20.4)$ & $<0.01$ \\
\hline Endpoint after presentation (all patients) & $37(4.7)$ & $57(7.5)$ & $92(9.6)$ & $<0.01$ \\
\hline Endpoint after presentation for patients without index ACS (\% of patients without index ACS) & $23(3.4)$ & $35(5.5)$ & $52(6.8)$ & 0.36 \\
\hline Endpoint after presentation for patients with index ACS (\% of patients with index ACS) & $14(13.7)$ & $22(18.03)$ & $40(20.4)$ & 0.01 \\
\hline \multicolumn{5}{|l|}{ Risk factors } \\
\hline Hypertension & $383(48.5)$ & $373(48.8)$ & $445(46.3)$ & 0.49 \\
\hline Dyslipidaemia & $374(47.4)$ & $380(49.7)$ & $443(46.1)$ & 0.31 \\
\hline Diabetes & $97(12.3)$ & $110(14.4)$ & $125(13.0)$ & 0.46 \\
\hline Family history of CAD & $380(48.2)$ & $396(51.8)$ & $488(50.7)$ & 0.33 \\
\hline Current or recent smoker & $165(20.9)$ & $158(20.7)$ & $224(23.3)$ & 0.34 \\
\hline \multicolumn{5}{|l|}{ Cardiovascular history } \\
\hline Prior MI & $154(19.5)$ & $177(23.2)$ & $206(21.4)$ & 0.21 \\
\hline Prior Angina & $205(26.0)$ & $265(34.7)$ & $287(29.8)$ & $<0.01$ \\
\hline$C A D$ & $141(17.9)$ & $152(19.9)$ & $167(17.4)$ & 0.37 \\
\hline Prior CABG & $44(5.6)$ & $68(8.9)$ & $75(7.8)$ & 0.04 \\
\hline Prior angioplasty & $117(14.8)$ & $134(17.5)$ & $157(16.3)$ & 0.35 \\
\hline
\end{tabular}




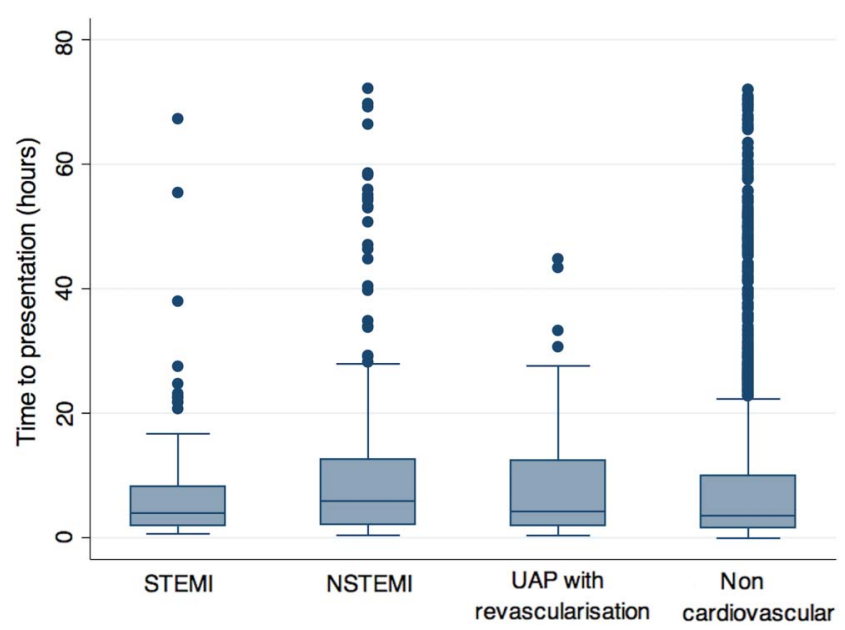

Figure 3 Delay time to presentation by index endpoint. Boxes represent 25th, 50th and 75th percentile while whiskers are upper and lower adjacent values. STEMI, ST-elevated myocardial infarct; NSTEMI, non-ST-elevated myocardial infarct; UAP, unstable angina pectoris.

\section{RESULTS}

The final enrolled sample included 3188 individuals. Thirty individuals were excluded for heart failure, 183 did not have data on time to presentation and 460 did not consent to 1 -year follow-up $(\mathrm{n}=460)$. The final sample included 2515 patients (figure 2); 1499 (59.6\%) were male and the mean age was

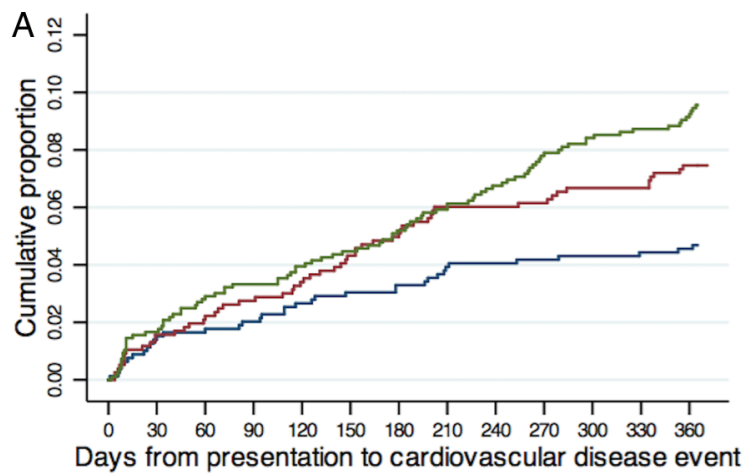
Number at risk

$\begin{array}{llllllllllllll}<2 \text { hours } & 789 & 778 & 776 & 773 & 768 & 765 & 763 & 758 & 757 & 756 & 755 & 754 & 753\end{array}$ $\begin{array}{llllllllllllll}2-6 \text { hours } & 764 & 752 & 748 & 743 & 738 & 731 & 726 & 718 & 718 & 717 & 713 & 713 & 707\end{array}$ $\begin{array}{llllllllllllll}>6 \text { hours } & 962 & 946 & 935 & 930 & 924 & 919 & 912 & 905 & 897 & 887 & 881 & 878 & 874\end{array}$

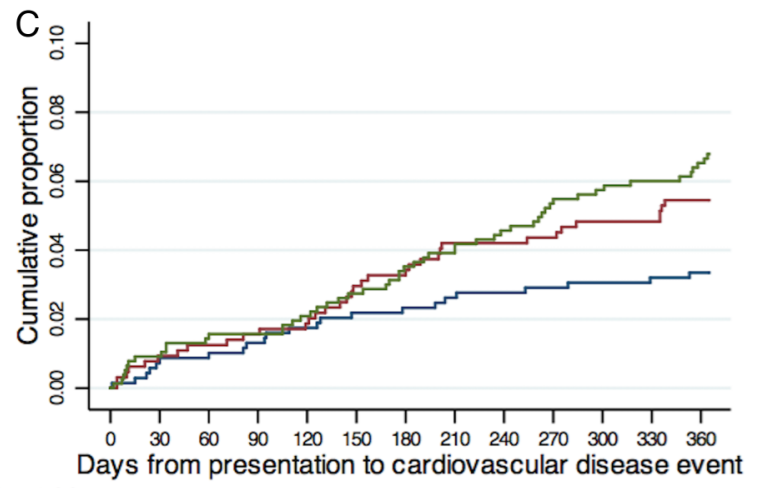

$\begin{array}{llllllllllllll}<2 \text { hours } & 687 & 682 & 681 & 678 & 675 & 672 & 671 & 669 & 668 & 667 & 666 & 665 & 664\end{array}$

$\begin{array}{llllllllllllll}2-6 \text { hours } & 642 & 636 & 634 & 632 & 630 & 623 & 621 & 615 & 615 & 614 & 611 & 611 & 607\end{array}$

$\begin{array}{llllllllllllll}>6 \text { hours } & 766 & 759 & 755 & 754 & 750 & 745 & 739 & 736 & 731 & 725 & 722 & 720 & 716\end{array}$
58 years $(\mathrm{SD}=15$, range $19-95)$. There was no loss to follow-up. Demographic characteristics of the sample by time to presentation are provided in table 1 . One-year endpoints occurred in $5.3 \%(110 / 2095)$ of patients without index ACS. One-year endpoints occurred after the index event in $18.1 \%$ of patients with an index diagnosis of ACS (76/420). Late presenters were slightly older and were more likely to be diagnosed with ACS during their index presentation compared with early or middle presenters. Early presenters were less likely to have had prior angina or have undergone coronary artery bypass graft (CABG) compared with those middle presenters and were more likely to be Caucasian compared with middle and late presenters.

Figure 3 provides further data on the time to presentation by index event. Median time to presentation was $3.53 \mathrm{~h}$ (IQR 1.55-9.86) for patients presenting with non-ACS diagnoses, $3.96 \mathrm{~h}$ (IQR 1.83-8.18) for those with STEMI, $4.22 \mathrm{~h}$ (IQR 1.97-12.33) for those with UAP treated with emergency or urgent revascularisation and $5.89 \mathrm{~h}$ (IQR 2.15-12.48) for those with NSTEMI. Time to presentation displayed right skew such that there were a number of individuals with long delays to presentation. Two hundred and eighty one patients (11.2\%) presented to ED $>24 \mathrm{~h}$ after the onset of symptoms.

Time to first endpoint was calculated for early, middle and late presenters. The log-rank test for the equality of survivor functions for early, middle and late presenters indicated that both late $(\mathrm{p}<0.01)$ and middle $(\mathrm{p}=0.03)$ presenters had higher rates of the primary endpoint than early presenters (figure 4). After adjustment for age, ethnicity, prior angina, prior CABG

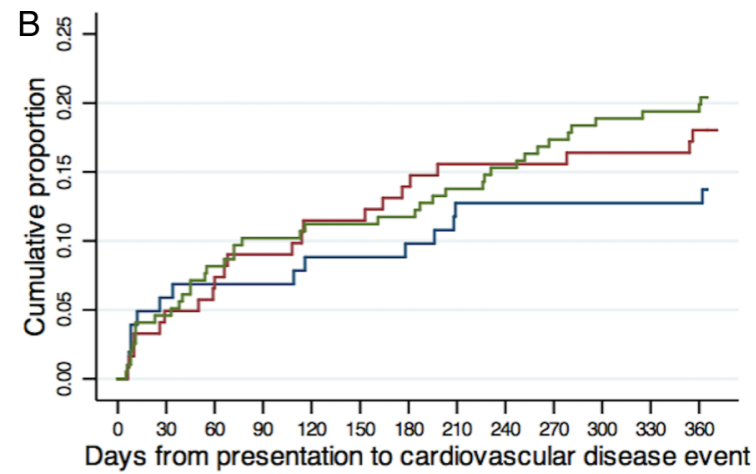
Number at risk

Figure 4 Time to primary endpoint for eary,
syndrome (ACS). (C) are those without ACS. 
and index ACS, the rate of the primary endpoints was 1.57 (95\% CI 1.07 to 2.31) times higher for late presenters compared with early presenters. Early and middle presenters had a similar rate of primary endpoints $(\mathrm{HR}=1.22,95 \% \mathrm{CI} 0.80$ to 1.85). As shown in table 2 , the rate of primary endpoints also was higher for patients with prior angina and older individuals but lower for Caucasian individuals.

Figure 4B, C provides the time to endpoint for ACS and non-ACS patients, respectively. A Cox regression model was conducted including age, ethnicity, angina, prior CABG, index ACS and the interaction between index ACS and time of arrival. Within this analysis, index ACS was associated with a higher rate of the primary endpoint $(\mathrm{HR}=2.81,95 \% \mathrm{CI} 1.44$ to 5.49$)$, but the interaction between ACS and time of arrival was not significant $(p=0.78)$ indicating that the relationship between time of arrival and the primary endpoint did not significantly differ across patients with and without index ACS. Table 2 provides estimates for the Cox regression models stratified by ACS. After controlling for age, prior angina, prior CABG and ethnicity, late presentation patients had slightly increased rate of primary endpoints compared with early presenters (HR for ACS $=1.38$ (95\% CI 0.75 to 2.54), HR for non-ACS 1.72 (95\% CI 1.05 to 2.82)). Overall, the Cox regression analyses on those individuals presenting with and without ACS revealed similar results to the total cohort. However, in the cohort of patients with index ACS, the sample size was smaller resulting in wider CIs. As such, the HRs for ethnicity and late presenters included one (were not significant).

The Cox regression analysis was repeated for the ACS cohort with index STEMIs excluded. Compared with early presenters, the primary endpoint occurred at a rate 1.41 (95\% CI 0.65 to 3.06 ) and 1.78 (95\% CI 0.88 to 3.58 ) times higher for middle and late presenters, respectively.

\section{DISCUSSION}

This is the first report that confirms an association between time to presentation and 12-month health outcomes in ED patients with symptoms of possible ACS in whom there was not an index ACS event. Longer time to presentation with symptoms of possible ACS was associated with increased rates of subsequent cardiac events, including death, ACS and readmission for heart failure that occurred up to 12 months after the index presentation. Irrespective of the cause for the index presentation, patients who presented within two hours of symptom onset had fewer cardiac events at 12 months. These results suggest that delay to reperfusion treatment in patients with STEMI and delay to presentation to ED in general is a marker of poor prognosis. Therefore, factors that contribute to a delay in seeking medical attention with symptoms of possible ACS are likely to contribute to an adverse prognosis in all patients regardless of the initial diagnosis. Although this study was not designed to investigate reasons for patient delay, it is possible that patients who have delayed seeking care for symptoms of chest discomfort may be reluctant to seek assistance for other medical conditions, or deny symptoms of other serious diseases. ${ }^{10} 112627$

Presenting to the ED with chest pain, irrespective of the cause of the symptom, is indicative of a risk for disease, with our study showing that after discharge from the index presentation, there was a high rate of adverse endpoints in patients with and without index ACS diagnoses. In those with a diagnosis of ACS during the initial hospital admission, the overall 1-year primary endpoint rate was $18.1 \%$. One-year endpoint rates for patients in whom there was not an initial diagnosis of ACS was significant $(5.3 \%)$, and is in keeping with previous reports showing that ED patients with a final diagnosis that was not ACS have adverse event rates of $>4 \%$ at 12 months, and have worse outcomes than that of age-matched populations. ${ }^{19} 2829$

A history of risk factors for cardiovascular disease or known cardiovascular disease, including previous myocardial infarction or PCI, was not associated with early $(<2 \mathrm{~h})$ presentation to hospital after the onset of symptoms of possible ACS. The reasons for this observation were not investigated in this study; however patients with known cardiovascular disease may have delayed seeking ED assessment only after unsuccessful trial of selftreatment. It remains important though to emphasise timeliness of medical assistance in educational programmes for high-risk patients, as such programmes aimed at the general population have shown reductions in time to presentation. ${ }^{13}$ Younger

Table 2 Cox proportional hazards model for all patients and for subgroups with and without index ACS

\begin{tabular}{|c|c|c|c|}
\hline & $\begin{array}{l}\text { HRs for all individuals } \\
(\mathrm{n}=2448)\end{array}$ & $\begin{array}{l}\text { HRs for individuals with index ACS } \\
(n=411)\end{array}$ & $\begin{array}{l}\text { HRs for individuals without index ACS } \\
(\mathrm{n}=2037)\end{array}$ \\
\hline Variable & HR $(95 \% \mathrm{Cl})$ & $\mathrm{HR}(95 \% \mathrm{Cl})$ & $\mathrm{HR}(95 \% \mathrm{Cl})$ \\
\hline \multicolumn{4}{|c|}{ Delay time (hours) } \\
\hline$<2$ & 1.00 & 1.00 & 1.00 \\
\hline $2-6$ & $1.22(0.80$ to 1.85$)$ & $1.07(0.54$ to 2.13$)$ & $1.30(0.77$ to 2.21$)$ \\
\hline$>6$ & 1.57 (1.07 to 2.31$)$ & $1.38(0.75$ to 2.54$)$ & $1.72(1.05$ to 2.82$)$ \\
\hline \multicolumn{4}{|l|}{ Age (years) } \\
\hline$<40$ & 1.00 & 1.00 & 1.00 \\
\hline $40-49$ & $1.04(0.32$ to 3.39$)$ & - & 0.70 (0.16 to 3.14$)$ \\
\hline $50-59$ & $2.18(0.76$ to 6.24$)$ & $1.14(0.42$ to 3.11$)$ & $2.04(0.59$ to 7.00$)$ \\
\hline $60-69$ & 2.62 (0.92 to 7.48$)$ & 0.51 (0.18 to 1.49$)$ & $3.72(1.11$ to 12.50$)$ \\
\hline $70-79$ & 4.37 (1.53 to 12.45$)$ & 1.32 (0.52 to 3.34$)$ & 5.43 (1.60 to 18.45$)$ \\
\hline$\geq 80$ & 6.36 (2.21 to 18.27$)$ & 2.87 (1.16 to 7.14$)$ & 5.49 (1.56 to 19.30$)$ \\
\hline Caucasian & $0.57(0.39$ to 0.83$)$ & 0.59 (0.32 to 1.08$)$ & $0.57(0.35$ to 0.93$)$ \\
\hline Prior angina & 2.98 (2.11 to 4.21$)$ & 1.99 (1.16 to 3.23$)$ & 3.91 (2.46 to 6.22$)$ \\
\hline Prior CABG & 1.03 (0.69 to 1.53$)$ & $1.14(0.60$ to 2.15$)$ & $1.00(0.60$ to 1.67$)$ \\
\hline Index ACS & 2.29 (1.69 to 3.10$)$ & & \\
\hline
\end{tabular}


patients in our study were more likely to present within $2 \mathrm{~h}$ of symptom onset, in keeping with previous findings. ${ }^{11}$ Reasons for delay were not specifically investigated in this study; however this finding may be due to anxiety caused by the symptom of chest pain, or by improved awareness of the warning signs for AMI in the general public.

Time to presentation to the ED did not correlate strongly with the underlying cause of the patients symptoms, although patients with a STEMI and those with non-cardiac chest pain were more likely to present in $<2 \mathrm{~h}$ than those with NSTEMI. While it is possible that STEMI causes severe symptoms and prompts patients to seek earlier medical care, there has been no research comparing the severity of symptoms for patients with STEMI versus NSTEMI. This study is unable to identify why patients without ACS present early to the ED.

There are two limitations of note. First, the ability of patients to gauge and accurately recall the time of symptom onset may have led to inaccuracies in the time reported and this inaccuracy may not be linear in nature. Second, presentation times do not account for those who presented to their primary care providers initially, which may have falsely elevated their time to presentation. This could be addressed in future prospective studies which collect the requisite data to account for primary care presentations.

\section{CONCLUSION}

We found an association between time to presentation and 1-year cardiac outcomes including death, AMI, UAP treated with emergency or urgent revascularisation or readmission with heart failure following initial assessment for ED patients with possible cardiac chest pain in the Australian and New Zealand setting irrespective of the eventual diagnosis. Effective public health campaigns, and other measures, that facilitate early presentation by explicitly encouraging patients to seek early medical attention with symptoms suggestive of ACS are justified and have the potential to improve prognosis.

Twitter Follow John Pickering at @kiwiskiNZ

Acknowledgements We acknowledge and thank the patients who participated in the study, and the research staff, ED staff and laboratory technicians of both participating sites for their invaluable efforts.

Contributors LC: study design, data interpretation, literature search, writing, reviewing, editing. JHG: study design, data collection, data interpretation, literature search, writing, reviewing, editing. LM: literature review reviewing editing. AL: literature review reviewing editing. MT: study design, data interpretation, writing, reviewing, editing. CP: data interpretation reviewing and editing. SA: data interpretation, reviewing. JP: data interpretation, reviewing, editing. ED: reviewing, editing, manuscript preparation. BC: reviewing and editing. RF: reviewing and editing. WAP: study design, data interpretation, literature search, writing, reviewing, editing.

Funding This study was funded by the Heart Foundation of Australia. LC is supported by the Queensland Emergency Medicine Research Foundation as the Noel Stevenson Fellow.

Competing interests None declared.

Ethics approval Royal Brisbane and Women's Hospital HREC.

Provenance and peer review Not commissioned; externally peer reviewed.

\section{REFERENCES}

1 Swanson N, Montalescot G, Eagle KA, et al. Delay to angiography and outcomes following presentation with high-risk, non-ST-elevation acute coronary syndromes: results from the Global Registry of Acute Coronary Events. Heart 2009;95:211-15.

2 Graff LG, Wang Y, Borkowski B, et al. Delay in the diagnosis of acute myocardial infarction: effect on quality of care and its assessment. Acad Emerg Med 2006;13:931-8.

3 Sorajja P, Gersh BJ, Cox DA, et al. Impact of delay to angioplasty in patients with acute coronary syndromes undergoing invasive management: analysis from the ACUITY (Acute Catheterization and Urgent Intervention Triage strategY) trial. J Am Coll Cardiol 2010;55:1416-24.
4 Ting $H H$, Bradley $E H$, Wang $Y$, et al. Delay in presentation and reperfusion therapy in ST-elevation myocardial infarction. Am J Med 2008;121:316-23.

5 Bruins Slot MH, Rutten FH, van der Heijden GJ, et al. Gender differences in pre-hospital time delay and symptom presentation in patients suspected of acute coronary syndrome in primary care. Fam Pract 2012;29:332-7.

6 Kostapanos MS, Florentin M, Elisaf MS. Gender Differences in the Epidemiology, Clinical Presentation, Prevention, and Prognosis of Acute Coronary Syndromes. Angiology 2013:64:5-8.

7 Acharya P, Adhikari RR, Bhattarai J, et al. Delayed presentation of acute coronary syndrome: a challenge in its early management. JNMA I Nepal Med Assoc 2009:48:1-4.

8 Darling C, Saczynski JS, McManus DD, et al. Delayed hospital presentation in acute decompensated heart failure: clinical and patient reported factors. Heart Lung 2013;42:281-6

9 Garofalo D, Grey C, Lee $M$, et al. Pre-hospital delay in acute coronary syndromes: PREDICT CVD-18. N Z Med J 2012;125:12-22.

10 Kirchberger I, Heier M, Wende R, et al. The patient's interpretation of myocardial infarction symptoms and its role in the decision process to seek treatment: the MONICA/KORA Myocardial Infarction Registry. Clin Res Cardiol 2012;101:909-16.

11 McKee G, Mooney M, O'Donnell S, et al. Multivariate analysis of predictors of pre-hospital delay in acute coronary syndrome. Int I Cardiol 2013;168:2706-13.

12 Mooney M, McKee G, Fealy G, et al. A randomized controlled trial to reduce prehospital delay time in patients with acute coronary syndrome (ACS). J Emerg Med 2014:46:495-506.

13 Naegeli B, Radovanovic D, Rickli H, et al. Impact of a nationwide public campaign on delays and outcome in Swiss patients with acute coronary syndrome. Eur $\int$ Cardiovasc Prev Rehabil 2011;18:297-304

14 McCullough PA, Gibson CM, Dibattiste PM, et al. Timing of angiography and revascularization in acute coronary syndromes: an analysis of the TACTICS-TIMI-18 trial. J Interv Cardiol 2004;17:81-6.

15 Pitsavos C, Kourlaba G, Panagiotakos DB, et al. Factors associated with delay in seeking health care for hospitalized patients with acute coronary syndromes: the GREECS study. Hellenic J Cardiol 2006:47:329-36.

16 Doyle F, De La Harpe D, McGee H, et al. Nine-year comparison of presentation and management of acute coronary syndromes in Ireland: a national cross-sectional survey. BMC Cardiovasc Disord 2005;5:5

17 De Luca G, Suryapranata $\mathrm{H}$, Ottervanger JP, et al. Time delay to treatment and mortality in primary angioplasty for acute myocardial infarction: every minute of delay counts. Circulation 2004;109:1223-5.

18 Elbarouni B, Goodman SG, Yan RT, et al. Impact of delayed presentation on management and outcome of non-ST-elevation acute coronary syndromes. Am Heart J 2008;156:262-8.

19 Geraldine McMahon C, Yates DW, Hollis S. Unexpected mortality in patients discharged from the emergency department following an episode of nontraumatic chest pain. Eur J Emerg Med 2008;15:3-8.

20 Than M, Cullen L, Aldous S, et al. 2-Hour accelerated diagnostic protocol to assess patients with chest pain symptoms using contemporary troponins as the only biomarker: the ADAPT trial. I Am Coll Cardiol 2012:59:2091-8.

21 Luepker RV, Apple FS, Christenson RH, et al. Case definitions for acute coronary heart disease in epidemiology and clinical research studies: a statement from the AHA Council on Epidemiology and Prevention; AHA Statistics Committee; World Heart Federation Council on Epidemiology and Prevention; the European Society of Cardiology Working Group on Epidemiology and Prevention; Centers for Disease Control and Prevention; and the National Heart, Lung, and Blood Institute. Circulation 2003:108:2543-9.

22 Pollack CV Jr, Antman EM, Hollander JE. 2007 focused update to the ACC/AHA guidelines for the management of patients with ST-segment elevation myocardial infarction: implications for emergency department practice. Ann Emerg Med 2008:52:344-55.e1.

23 Cullen $\mathrm{L}$, Than M, Brown AF, et al. Comprehensive standardized data definitions for acute coronary syndrome research in emergency departments in Australasia. Emerg Med Australas 2010;22:35-55.

24 Thygesen $\mathrm{K}$, Alpert JS, Jaffe AS, et al. Third universal definition of myocardial infarction. Circulation 2012;126:2020-35.

25 Pocock S, Clayton T, Altman D. Survival plots of time-to-event outcomes in clinical trials: good practice and pitfalls. Lancet 2002;359:1686-9.

26 Meischke $\mathrm{H}, \mathrm{Ho}$ MT, Eisenberg MS, et al. reasons patients with chest pain delay or do not call 911. Ann Emerg Med 1995;25:193-7.

27 Cha KS, Park JS, Ahn J, et al. Non-chest pain complaint at presentation delays door-to-balloon time and are associated with worse clinical outcomes in patients with ST-segment elevation myocardial infarction. J Am Coll Cardiol 2014;63.

28 Prina LD, Decker WW, Weaver AL, et al. Outcome of patients with a final diagnosis of chest pain of undetermined origin admitted under the suspicion of acute coronary syndrome: a report from the Rochester Epidemiology Project. Ann Emerg Med 2004:43:59-67.

29 Manini AF, Gisondi MA, van der Vlugt TM, et al. Adverse cardiac events in emergency department patients with chest pain six months after a negative inpatient evaluation for acute coronary syndrome. Acad Emerg Med 2002;9:896-902. 